\title{
Application of the Multilevel Matrix Decomposition Algorithm to the Frequency Analysis of Large Microstrip Antenna Arrays
}

\author{
Josep Parrón, Juan M. Rius, Member, IEEE, and Juan R. Mosig, Fellow, IEEE
}

\begin{abstract}
The application of integral equation methods based in the method of moments discretization to solve large antenna arrays is difficult due to the fact that the computational requirements increase rapidly with the number of unknowns. This is critical when a frequency analysis of the antenna is required. We propose the multilevel matrix decomposition algorithm (MLMDA) to carry out this purpose efficiently. As the MLMDA method is particularly well-suited for the analysis of planar structures with any Green's function, it is a very efficient approach for the frequency analysis of microstrip antenna arrays.
\end{abstract}

Index Terms-Antenna arrays, integral equations, iterative methods, microstrip, moment methods.

\section{INTRODUCTION}

$\mathbf{T}$ HE APPLICATION of integral equation (IE) methods [1] based on the method of moments (MoM) discretization [2] to the analysis of three-dimensional (3-D) electrically large arbitrary surfaces is difficult due to the rapid increase of the computational requirements with the number of unknowns, $N$, (roughly as $N^{2}$ the storage memory and $N^{3}$ the operation count in the direct solution), so when trying to solve problems of the order of a few thousand unknowns, the capability of our personal computer is easily overcome. The use of techniques based on the iterative solution of the system of equations like the generalized minimum residual (GMRES) method can partially mitigate this problem by greatly reducing the time required to solve the linear system. In this case, it is necessary to compute efficiently the matrix-vector products appearing in iterative methods. Linear system solution time becomes critical when the antenna parameters have to be computed for many operating frequencies.

One of the most techniques that exploit the physical or mathematical properties of the impedance matrix to perform an efficient matrix-vector product is the multilevel matrix decomposition algorithm (MLMDA), which was initially proposed for two dimensional objects in [3]. The extension of this algorithm

Manuscript received July 5, 2001; revised October 25, 2001. This work was supported by the European Commission, the "Departament d'Universitats Recerca i Societat de la Informació (DURSI)" of the "Generalitat de Catalunya and the Spanish "Comisión Interministerial de Ciencia y Tecnología (CICYT)" under Grants FEDER 2FD97-0135 and TIC 98-1037. The work of J. M. Ruis was supported in part by EPFL.

J. Parrón and J. M. Rius are with the Signal Theory and Communication Group, Universitat Politècnica de Catalunya, 08034 Barcelona, Spain (e-mail: rius@ tsc.upc.es).

J. R. Mosig is with the Laboratory of Electromagnetics and Acoustics, École Politechnique Fédérale de Lausanne, CH1015 Lausanne, Switzerland (e-mail Juan.Mosig@epfl.ch).

Publisher Item Identifier S 0018-9464(02)02439-1. to arbitrary 3-D surfaces [4] showed that it is particularly efficient for the analysis of planar structures of arbitrary shape discretized into Rao, Wilton, and Glisson (RWG) linear triangle basis functions [5].

In this approach, special Green's functions, such as the multilayer media Green's function, are very easy to implement [6]. In fact, the MLMDA can be used at the top of any existing MoM code. This feature, together with the good performance of the algorithm for piecewise planar objects, makes the MLMDA more suitable for the frequency analysis of large printed antenna arrays than other existing methods.

\section{THE ALGORITHM}

The electric field integral equation (EFIE) in the frequency domain discretized by MoM may be expressed in matrix form as [1], [2]

$$
-\left[E^{i}\right]=[Z][J]
$$

where $[J]$ are the coefficients of the induced current discretized in RWG basis functions (unknown), $[Z]$ is the impedance matrix and $\left[E^{i}\right]$ the discretization of the incident field.

Induced current coefficients $[J]$, can be obtained solving iteratively (1) (e.g., using GMRES). In each iteration, the main computational effort to obtain the $k$ th estimation of the induced current $\left[J^{(k)}\right]$ is the matrix-vector product $[Z]\left[J^{(k-1)}\right]$. Using direct matrix-vector multiplication, the operation count and the memory requirements for each iteration are proportional to $N^{2}$, if $N$ is large.

\section{A. Multilevel Subdivision of the Object}

In order to reduce the huge operation count in the direct matrix-vector multiply, let us divide the object into many non overlapping boxes as shown in Fig. 1. Then, the matrix-vector (1) can be computed by adding, for each pair of boxes, the field due to the RWG basis functions at source box tested by the RWG weighting functions at the observation box

$$
\left[E_{m}\right]=\left[Z_{m n}\right]\left[J_{n}\right]
$$

where $n$ and $m$ respectively refer to the indices of the RWG basis functions in the source and observation box and $\left[Z_{m n}\right]$ is a submatrix of the impedance matrix. Let us denote as $S_{s}$ and $S_{o}$ the size of the source and observation box, respectively, while $d$ is the distance between the centers of the boxes. We also 


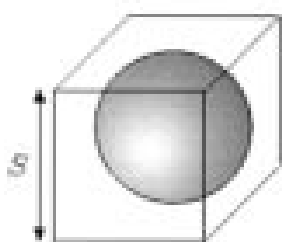

Level 0

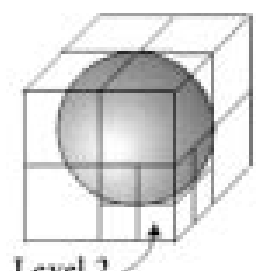

Level 2 .
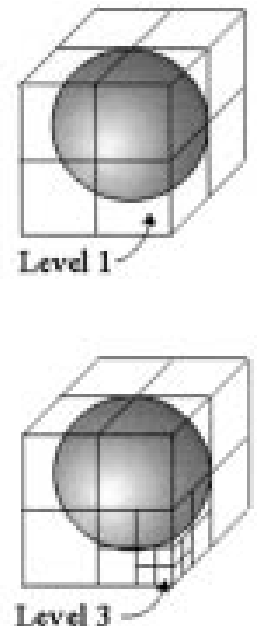

Level 3
Level 1

Fig. 1. Multilevel subdivision of the box enclosing the object.

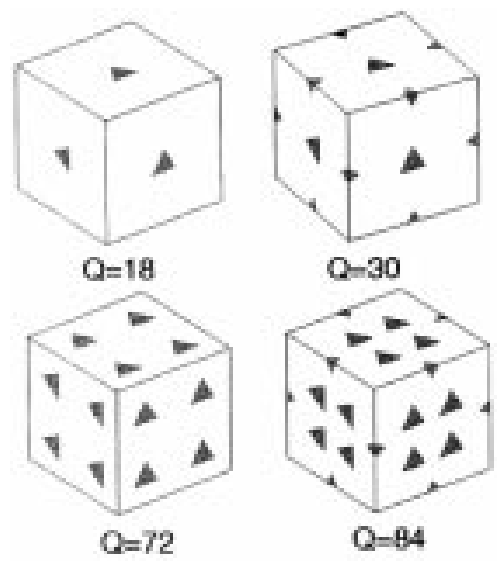

Fig. 2. Equivalent RWG functions defined for a box are evenly distributed in faces and edges.

consider that there are $N_{n}$ original RWG functions in the source box and $N_{m}$ in the observation box.

Hence, a recursive procedure, which will begin at level 2 and will stop at the finest level $L$, is introduced. For a couple of nonempty source and observation boxes which belong to the same subdivision level $l(2 \leq l \leq L)$, two cases are possible.

1) Boxes are Touching Each Other: Then they are subdivided into level $l+1$ boxes, until $l=L$. When $l=L$ is reached, apply direct multiplication (2) in $N_{n} N_{m}$ operations.

2) Boxes are Nontouching Each Other: Now, $\left[E_{m}\right]$ can be represented with a reduced number of degrees of freedom $Q$ since the rank of $\left[Z_{m n}\right]$ is much smaller than $N_{n}$ and $N_{m}$ [7], so (2) can be computed in much less than $N_{n} N_{m}$ operations using the schemes described in next sections. It is important to point out that $Q$ grows as $T^{0.4}$, where $T=\left(S_{s} S_{o}\right) /(d \lambda)$ and $\lambda$ is the wavelength [4], [6].

\section{B. Equivalent Functions}

In order to compute in an efficient way the interaction between two nontouching boxes at the same level some equivalent functions on the surface of each box are defined. In general, they must be located in the boundary of the box (Fig. 2). However, if all the original RWG basis functions in the box are contained

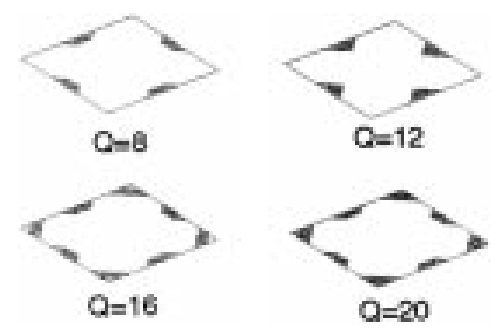

Fig. 3. Equivalent functions when all the original RWG are contained in a plane.

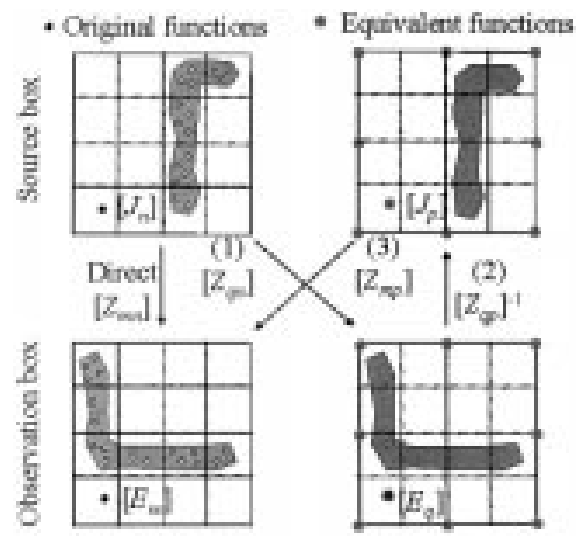

Fig. 4. MDA performed in three steps.

in a plane, they may be located in the boundary of the rectangle resulting from the intersection between the box and the plane (Fig. 3).

The number of equivalent functions must be equal or greater than $Q$. In [4] and [6], it can be seen that the required $Q$ is much smaller for the case of planar objects than for general 3-D objects. This implies that the following algorithms will be much more efficient for planar objects, an example of which are microstrip antennas.

\section{Matrix Decomposition Algorithm (MDA)}

Now, let us use $p$ and $q$ to refer to the indexes of the equivalent RWG functions at the boundary of the source and observation boxes. Then, we perform the matrix-vector multiplication in the following three steps (Fig. 4).

1) Compute the field due to the original RWG basis functions at source box tested by the equivalent RWG weighting functions at the observation box.

$$
\left[E_{q}\right]=\left[Z_{q n}\right]\left[J_{n}\right] .
$$

2) Find the equivalent functions at the source box that produce the same field $\left[E_{q}\right]$

$$
\left[J_{p}\right]=\left[Z_{q p}\right]^{-1}\left[E_{q}\right] .
$$

3) Compute the field at the original RWG in the observation box. The equivalent sources $\left[J_{p}\right]$ produce the same field as the original sources $\left[J_{n}\right]$

$$
\left[E_{m}\right]=\left[Z_{m p}\right]\left[J_{p}\right] .
$$




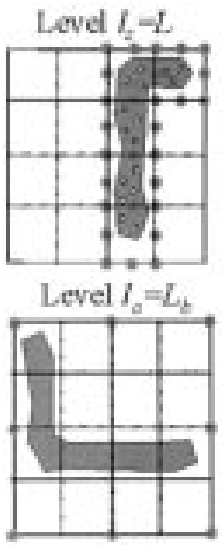

a)
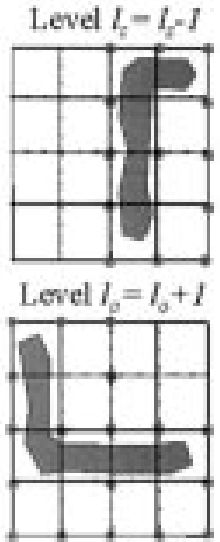

b)

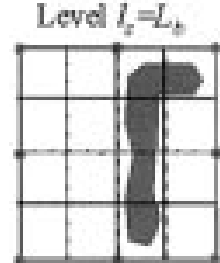

Level $l_{a}=L$

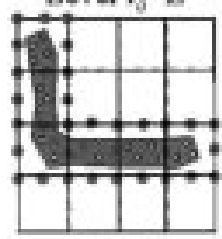

c)
Fig. 5. Multilevel matrix decomposition algorithm scheme.

Now, the operation count is proportional to

$$
Q N_{n}+c Q^{3}+Q^{2}+N_{m} Q
$$

where in our implementation $c$ is of the order of $1 / 500$. Since $Q$ is much smaller than $N_{n}$ and $N_{m}$ when we consider nontouching boxes, this alternative approach is much faster than the direct submatrix-vector product (2).

\section{Multilevel Matrix Decomposition Algorithm (MLMDA)}

In the analysis of electrically large objects, $Q$ can become very large for boxes in coarser levels. In that case another algorithm, as proposed in [3], can be used to improve the efficiency of the MDA. Fig. 5 shows that this algorithm can be understood as a multilevel version of MDA.

In practice, the MLMDA has a computational overload due to the generation and positioning of so many sets of equivalent functions, so the MLMDA only results more efficient than the MDA for very large boxes (side $>8 \lambda$ ).

\section{BENCHMARK}

As the MDA and the MLMDA do not rely on specific mathematical properties of the Green's functions, they can be applied to most commonly used configurations governed by special Green's functions like multilayered media. For this reason these algorithms are suitable for the frequency analysis of large micrsotrip antenna arrays. The algorithm has been implemented in MATLAB 5 [8] high-level programming language, except for the computation of impedance submatrices between subsets of RWG basis and testing functions, that were coded in C language in order to optimize the computing time. An AMD Athlon 1.33 $\mathrm{GHz}$ personal computer with $1536 \mathrm{MB}$ of RAM and $40 \mathrm{MB} / \mathrm{s}$ transfer speed hard disk has been used in the simulations.

As a benchmark we consider the $8 \times 8$ microstrip series-fed array of Fig. 6, which has been discretized in 15280 RWG unknowns in order to achieve accurate results for input impedance. It must be noted here that if only far field results are needed, a coarser discretization with 7320 unknowns can be used. In Fig. 7 the measured reflection coefficient [9] is compared with the reflection coefficient computed using MLMDA. It can be seen as the agreement is excellent.

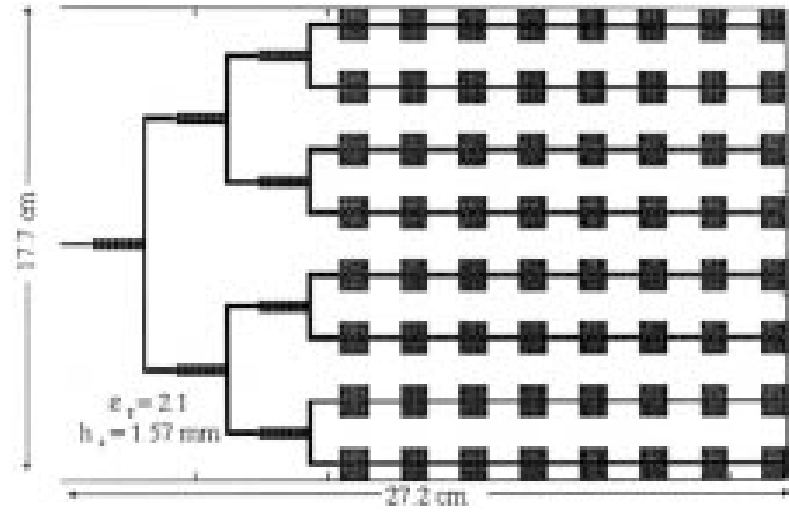

Fig. $6.8 \times 8$ microstrip series-fed array.

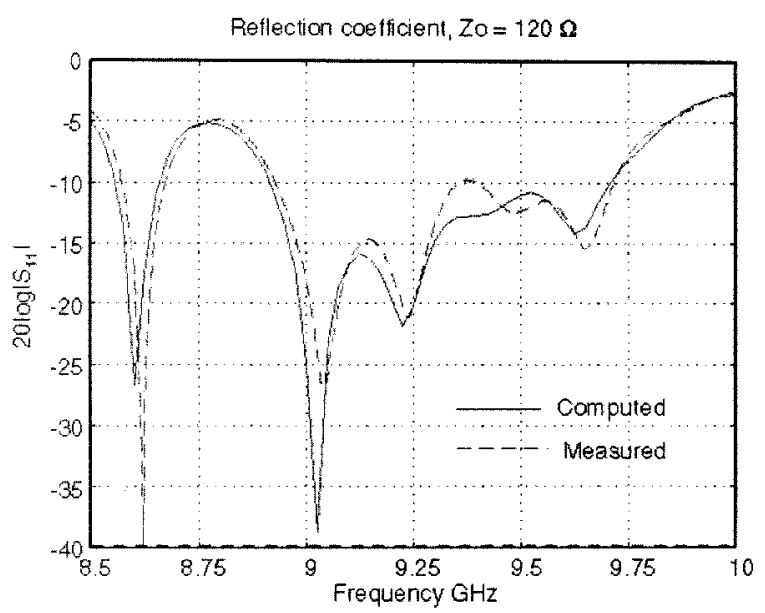

Fig. 7. Frequency analysis for $8 \times 8$ microstrip series-fed array.

The average computational requirements per frequency of MLMDA are the following for a smallest level box size of $0.4 \lambda$.

1) In order to reduce the number of iterations of GMRES, preconditioning is used (incomplete LU factorization [10]), taking $32 \mathrm{MB}$ of memory and $24 \mathrm{~s}$.

2) Interaction matrices between boxes are computed before the iteration procedure and saved in memory, requiring $341 \mathrm{MB}$ and $3 \mathrm{~m} 8 \mathrm{~s}$. In cases where there is not enough RAM to store interaction matrices, our implementation allows the use of hard disk and matrix elements recomputation, so that the solution of huge problems involving hundreds of thousands of unknowns is possible.

3) The GMRES algorithm converges to a relative residual error of $10^{-3}$ in 10 iterations, taking $9.7 \mathrm{~s}$ per iteration.

4) Total computation time is $6 \mathrm{~m} 9 \mathrm{~s}$ per frequency for the 15280 unknowns discretization used for the input reflection coefficient computation in Fig. 7.

In [11], we can find the computational requirements for the same geometry and residual error (which was defined as the square of the residual used in our implementation) at $9.42 \mathrm{GHz}$ using a BiCG + FFT solution. It required a regular mesh of 118073 rooftop basis functions and took $1 \mathrm{~h} 50 \mathrm{~m}$ using $18 \mathrm{MB}$ of memory in a DEC Alpha computer. As preconditioning is difficult to use with this method due to the large number of unknowns, 599 iterations were necessary to converge to the de- 
sired residual. Thus, the bottleneck of BiCG + FFT is the high number of iterations required as the number of unknowns grows.

It is quite difficult to perform a strict comparison between BiCG + FFT and BiCG + MLMDA, since in [11] there is not enough information about the DEC Alpha computer used. For the example presented in [11], the MLMDA scheme needs a 7320 unknowns discretization for accurate radiation pattern computation that is solved in $1 \mathrm{~m} 59 \mathrm{~s}$ using $184 \mathrm{MB}$ of memory to store the preconditioner and the interaction matrices.

Furthermore, the MLMDA can be always optimized to take advantage of all the available memory to achieve faster results.

\section{CONCLUSION}

Since the MLMDA does not rely on specific mathematical properties of the Green's functions, it is suitable for the analysis of microstrip structures. The MLMDA has been used in the frequency analysis of large microstrip antenna arrays, solving a problem of about 15000 unknowns in a personal computer. Reflection coefficient results agree very well with measurements. Compared to another method based in BiCG + FFT, the MLMDA achieves faster results with reasonable memory requirements. Besides, MLMDA scheme is very efficient and flexible, and can be easily optimized to make use of all the capabilities of the computer hardware to obtain the fastest possible results.

\section{REFERENCES}

[1] N. Morita, N. Kumagai, and J. R. Mautz, Integral Equation Methods for Electromagnetics. Boston, MA: Artech House, 1990.

[2] R. F. Harrington, Field Computation by Moment Methods. New York: MacMillan, 1968.

[3] E. Michelsen and A. Boag, "A multilevel matrix decomposition algorithm for analyzing scattering from large structures," IEEE Trans. Antennas Propagat., vol. 44, pp. 1086-1093, Aug. 1996.

[4] J. M. Rius, J. Parrón, E. Úbeda, and J. R. Mosig, "Multilevel matrix decomposition algorithm for analysis of electrically large electromagnetic problems in 3-D," Microwave Opt. Technol. Lett., vol. 22, no. 3, pp. 177-182, August 1999.

[5] S. M. Rao, D. R. Wilton, and A. W. Glisson, "Electromagnetic scattering by surfaces of arbitrary shape," IEEE Trans. Antennas Propagat., vol. 30, pp. 409-418, May 1982.

[6] J. Parrón, J. M. Rius, A. Heldring, E. Úbeda, and J. R. Mosig, "Analysis of microstrip antennas by multilevel matrix decomposition algorithm," in Proc. Euro. Congress Computational Methods Applied Sciences in Engineering, Barcelona, Spain, Sept. 2000.

[7] O. M. Bucci and G. Francescetti, "On the degrees of freedom of scattered fields," IEEE Trans. Antennas Propagat., vol. 37, pp. 918-926, July 1989.

[8] The Math Works Inc., Using Matlab Natick, MA, 1997.

[9] E. Suter, "Efficient numerical modeling of large scale planar antennas using a subdomain multilevel approach," Ph.D. dissertation, Ecole Polytechnique Federale de Lausanne, Lausanne, Switzerland, Sept. 2000.

[10] Y. Saad, Iterative Methods for Sparse Linear Systems. Boston, MA: PWS, 1996.

[11] C. F. Wang, F. Ling, and J. M. Jin, "A fast full-wave analysis of scattering and radiation from large finite arrays of microstrip antennas," IEEE Trans. Antennas Propagat., vol. 46, pp. 409-418, Oct. 1998. 